\title{
Generation of integration-free neural progenitor cells from cells in human urine
}

\author{
Lihui Wang1-3, Linli Wang1,2, Wenhao Huang1,2, Huanxing Su1,2,7, Yanting Xue1,2,4, \\ Zhenghui Su1,2,4, Baojian Liao1,2, Haitao Wang1,2, Xichen Bao1,2, Dajiang Qin1,2, Jufang \\ Hes, Wutian Wu6, Kwok Fai So6, Guangjin Pan1,2 \& Duanqing Pei1,2
}

1Key Laboratory of Regenerative Biology, South China Institute for Stem Cell Biology and Regenerative Medicine, Guangzhou Institutes of Biomedicine and Health, Chinese Academy of Sciences, Guangzhou, China. 2Guangdong Provincial Key Laboratory of Stem Cell and Regenerative Medicine, South China Institute for Stem Cell Biology and Regenerative Medicine, Guangzhou Institutes of Biomedicine and Health, Chinese Academy of Sciences, Guangzhou, China. 3Department of Pathology, Dalian Medical University, Dalian, China. 4School of Life Sciences, University of Science and Technology of China, Hefei, Anhui, China. 5Department of Rehabilitation Science, Hong Kong Polytechnic University, Hong Kong, China. 6Department of Anatomy, Hong Kong University, Hong Kong, China. 7Present address: State Key Laboratory of Quality Research in Chinese Medicine and Institute of Chinese Medical Sciences, University of Macau, Macao, China. Correspondence should be addressed to G.P. (pan_guangjin@ gibh.ac.cn) or D.P. (pei_duanqing@gibh.ac.cn).

Human neural stem cells hold great promise for research and therapy in neural disease. We describe the generation of integration-free and expandable human neural progenitor cells (nPcs). We combined an episomal system to deliver reprogramming factors with a chemically defined culture medium to reprogram epithelial-like cells from human urine into nPcs (huinPcs). these transgene-free huinPcs can self-renew and can differentiate into multiple functional neuronal subtypes and glial cells in vitro. Although functional in vivo analysis is still needed, we report that the cells survive and differentiate upon transplant into newborn rat brain.

Several neural disorders have no effective drug treatment at present, and stem cells offer hope for those suffering from these debilitating diseases. There has been intense interest in obtaining human neural stem cells (NSCs) that may be used to treat neural disorders or to study them in the laboratory. The isolation and use of NSCs from either fetal or adult human tissue remain challenging because of ethical concerns and immune rejection1,2. Induced pluripotent stem cell (iPSC) technology provides a promising solution to this problem because it may be used to generate patient-specific cells for autologous engraftment3-6. Indeed, iPSCs from patients with neural diseases have been established successfully, such as with Parkinson's disease7,8, Huntington's disease7 and Alzheimer's disease9. Differentiation of such iPSCs along the neural lineage and modeling of disease in vitro could increase our mechanistic understanding of these diseases, enable drug screening and eventually provide functional cells for autologous transplantation. However, the differentiation of human iPSCs into NSCs is inefficient, time consuming and variable among different iPSC lines6. Moreover, pluripotent cells such as iPSCs pose the risk of teratomas when transplanted in vivo. To attempt to circumvent these problems, new approaches have been devised to convert one somatic cell type to another without full reprogramming to the pluripotent state. Notably, functional neurons, termed induced neurons, 
have been generated directly from fibroblasts by retroviral delivery of neural-specific transcription factors or microRNAs10-15. The direct conversion approach has also been successfully applied to reprogram mouse fibroblasts into hepatocyte-like cells 16,17, cardiomyocytes17 and pancreatic $\beta$ cells 18 , using virally delivered reprogramming factors.

An attractive alternative to using induced neurons or iPSC-derived neurons is to generate self-renewable NPCs from a patient's own somatic cells, and to do so avoiding viral integration of the genes encoding reprogramming factors. In this report, we describe the integration-free generation of hUiNPCs, human NPCs from epithelial-like cells in human urine. The cells could be expanded in vitro and differentiate into neuronal subtypes and astrocytes.

\section{RESULTS}

\section{Generation of integration-free NPCs from human urine cells}

We have recently shown that human urine contains live cells that can be efficiently reprogrammed into iPSCs19. In an effort to further improve this approach to derive naive iPSCs, we adopted integration-free and feeder-free methods to reprogram human urine cells (HUCs). We purified viable HUCs from the urine (Fig. 1a) of a healthy 37-year-old male donor and transfected them with oriP/EBNA episomal vectors carrying a combination of reprogramming factors encoded by OCT4 (POU5F1), SOX2, SV40LT, KLF4 and microRNA cluster MIR302-367 through electroporation20,21 and cultured the transfected cells in defined basal medium containing FGF2 (ref. 22) and a cocktail of small molecules (5i) known to promote reprogramming: CHIR99021, PD0325901, A83-01, thiazovivin and DMH1 (refs. 23-29). We observed that the transfected HUCs showed rapid morphological changes in these cultures. Notably, as early as day 12 post-electroporation, we began to observe the formation of compact colonies (Fig. 1b). The average colony-forming efficiency was around $0.2 \%$ as determined from the initial number of HUCs used in each reprogramming experiment and was consistent for three different donors $(\mathrm{n}=$ 3, Supplementary Fig. 1). This is in sharp contrast to HUCs electroporated with the same episomal vectors but cultured in TeSR22, in which iPSC colonies appeared at around $25 \mathrm{~d}$ after electroporation (Supplementary Fig. 2). We were intrigued by the appearance of these early colonies and characterized them further.

We picked colonies from both TeSR and 5i cultures and cultured them further on Matrigel, in their original media. Surprisingly, the domed colonies that had emerged in the 5i medium assumed a rosette-like morphology typical of NPCs (Fig. 1c). In contrast, colonies grown in TeSR medium grew as typical human iPSC colonies (Supplementary Fig. 2). We hypothesized that cells undergoing reprogramming in the $5 \mathrm{i}$ medium might preferentially commit to the NPC fate at an early stage (around day 12) before the establishment of a full pluripotent state (around day 25). To test this hypothesis, we randomly picked 20 individual colonies from the reprogramming culture in $5 \mathrm{i}$ at day 14 or 15 and examined the expression of NSC and pluripotency markers without further re-plating or culturing. By quantitative real-time PCR (qRT-PCR) analysis, we observed robust expression of typical NSC genes such as SOX2 and NES (encoding nestin) in all 20 colonies. We did not observe substantial activation of genes encoding pluripotency markers such as OCT4 and NANOG or other lineage markers such as T (brachyury) and SOX17 (Fig. 1d and Supplementary Fig. 2). We did detect low expression of SOX17 in HUCs and some picked colonies, which is consistent with the endodermal origin of HUCs (Supplementary Fig. 2). PAX6, 
another NSC gene, was activated at day 14 , but more substantially so at day 15 , which suggests a dynamic process of reprogramming toward a neural fate (Fig. 1d).

We further immunostained the $5 \mathrm{i}$ colonies at day 15 for known embryonic stem cell (ESC) or NSC markers. The colonies were negative for pluripotency markers such as TRA-1-60, TRA-1-81 and NANOG but stained positively for PAX6 and nestin (Fig. 1e-i). We also failed to detect substantial activation of OCT4 and NANOG at earlier stages of reprogramming (days 6-8) (Supplementary Fig. 2). It has been reported that mouse embryonic fibroblasts reprogrammed with standard pluripotency factors (Oct4, Sox2, Klf4 and c-Myc) can be switched to a neural identity by transferring the virally infected cells to neural medium containing fibroblast growth factors and epidermal growth factor30. However, we did not observe NPC-like colonies among the transfected HUCs grown in the identical neural medium reported earlier (data not shown). Furthermore, when we replaced HUCs with human dermal fibroblasts, we observed no NPC-like cells under the conditions that we used for HUC reprogramming with 5i (data not shown). Taken together, our results suggest that the compact, dome-shaped colonies that appeared early during reprogramming in $5 \mathrm{i}$ medium may have committed to an NPC fate rather than a pluripotent one. We observed the same outcome for HUCs donated by two additional individuals ages 10 and 25 at an average reprogramming efficiency of around $0.2 \%$ based on the starting number of HUCs (Supplementary Fig. 1).

To characterize the proliferative potential of hUiNPCs, we dissociated the re-plated rosette colonies and seeded them as single cells on Matrigel (Fig. 1j). We observed that these cells grew well and exhibited typical NPC morphology. When cultured in suspension, the hUiNPCs grew as neural spheres, a typical property of NSCs31, and could be expanded over multiple passages with a high proliferation rate (Fig. 1k). The cells expressed high levels of the proliferation marker Ki67 (Fig. 11). hUiNPC spheres expanded for 11 passages also showed homogenous expression (>90\% of cells) of the neural ectoderm transcription factors PAX6 (ref. 32) and SOX1 as well as the neural stem cell markers SOX2 and nestin (Fig. 1m-o and Supplementary Fig. 2). In contrast, we did not detect the expression of pluripotency markers such as OCT4 or markers for other germ layer lineages such as SOX17 and T (Fig. 1p,q and Supplementary Fig. 2). The expanded hUiNPCs at passage 6 no longer harbored the reprogramming factors encoded by OCT4, SOX2, KLF4, SV40LT or MIR302-367, nor did they carry the genes from the episomal vector backbone (Fig. 1r), yet they possessed normal karyotypes (Fig. 1s). We also performed whole-genome sequencing of select hUiNPCs and confirmed that they are of human origin (data not shown).

\section{hUiNPCs show distinct gene expression profiles}

We profiled the global gene expression patterns of hUiNPCs, the parental HUCs and the urine cell-derived iPSCs (Uri_iPSCs). The hUiNPCs clustered independently from the starting HUCs (UriC1 and UriC2) and the urine iPSCs (Uri_iPSC1, Uri_iPSC2) (Fig. 2a). Further paired-comparison analysis revealed distinct expression profiles between hUiNPCs and Uri_iPSCs derived from the same starting cells: neural genes were more highly expressed in hUiNPCs, and pluripotency genes were more highly expressed in Uri_iPSCs (Fig. 2b). By comparing the expression profiles between HUCs and hUiNPCs, we identified 720 genes related to neuronal function that were upregulated in hUiNPCs (Fig. 2c,d and Supplementary Table 1). In contrast, the HUC expression profiles were enriched for genes related to endothelial, angiogenic or epithelial functions (Fig. 2c,d and Supplementary Table 2), indicating that the HUCs might be of endothelial or epithelial origin. 


\section{hUiNPCs differentiate into cell subtypes of neural lineage}

The promise of NPCs relies on their capacity to differentiate into functional subtypes of neural cells (Fig. 3). We observed that hUiNPCs (at passage 5) could efficiently give rise to $\beta$ III tubulin (TUJ1)-positive neurons (at an average efficiency of $76.7 \%$ of total cells in three experiments) and glial fibrillary acid protein (GFAP)-positive astrocytes (at an average efficiency of 7.8\% of total cells in three experiments) upon in vitro spontaneous differentiation (Fig. 3a,b,l). Moreover, this differentiation potential was well maintained throughout prolonged in vitro expansion (passage 8) (Fig. 31). Unlike mouse NPCs, human NPCs do not give rise to oligodendrocytes during spontaneous in vitro differentiation33,34. However, in the presence (for 3 weeks) of factors (PDGF-AA, NT3, IGF1) known to promote oligodendrocyte differentiation, we observed O4- and PDGFR- $\alpha-$ positive oligodendrocyte-like cells (Fig. 3h,i). We observed, by staining with neuronal subtype-specific markers, that hUiNPCs could generate various subtypes of neurons including mature glutamatergic, GABAergic and dopaminergic neurons at $37.6 \%, 15.2 \%$ and $6.5 \%$, respectively (Fig. 3e-g, respectively, and Fig. 3k). We also observed DCX-positive immature neurons in the differentiation culture (Fig. 3d). Finally, $85.4 \%$ of neurons derived from hUiNPC were positive for synapsin, indicating that most of the neurons were likely to be excitable (Fig. $3 \mathrm{j}, \mathrm{k})$.

\section{hUiNPC-derived neurons are functional in vitro}

We used standard whole-cell patch-clamp recordings to examine the function of hUiNPC-derived neurons (Fig. 4). We observed rapidly inactivating inward currents and persistent outward currents in response to depolarizing voltage steps that could be blocked by tetrodotoxin and tetraethylammonium (Fig. 4b,c). We also observed that the neurons generated repetitive trains of action potentials (Fig. 4a). Notably, the hUiNPC-derived neurons exhibited strong postsynaptic currents spontaneously or in response to excitatory or inhibitory neurotransmitter stimulation (Fig. $4 \mathrm{~d}-\mathrm{f} ; 5$ out of 5 neurons for each experiment). Together, these data demonstrate that hUiNPCs can give rise to mature, functional neurons invitro.

\section{Transplantation of hUiNPCs in vivo}

To examine the potential of hUiNPCs invivo, we transplanted these cells into the striatum of newborn rats $(\mathrm{n}=12$ rats) (Fig. 5a) and analyzed the brains of the animals 4 weeks after transplantation. We observed human nuclear antigen (hNA)-stained cells that had survived and migrated in the host brain (Fig. 5) and did not observe teratoma formation in any of the transplanted rats (Fig. 5a). Some hNA-positive cells apparently remained as NPCs, as they stained positively for the NSC marker nestin (Fig. 5b and Supplementary Fig. 3). We also observed hNA-positive cells that expressed the astrocyte marker GFAP and the neuronal marker TUJ1 (Fig. $5 b$ ), which indicated that the engrafted hUiNPCs could give rise to both neurons and astrocytes in vivo. A full characterization of the stability, differentiation potential and function of these cells in vivo will require further study.

\section{DISCUSSION}

We describe here the notable finding that functional NPCs can be generated from somatic cells with the same factors known to be capable of reprogramming to pluripotency when cells are cultured in specially defined conditions. Recently, several reports have shown that mouse fibroblasts can be reprogrammed directly into neural stem cells using virally delivered reprogramming factors35-38. In contrast, our method uses episomal factors for rapid and efficient 
derivation of integration-free NPCs from cells in human urine.

We showed that hUiNPCs arise at day 12-15, before the emergence of iPSCs at day 24-28 (Fig. 1). Further, the colonies express NSC markers, but we did not detect the expression of pluripotency markers (Fig. 1d-i). However, on the basis of our current data, which were obtained from mixed populations of cells, we cannot rule out the possibility that some cells in the reprogramming culture pass through a fully pluripotent state. Single-cell analysis will help clarify this question39,40 and may also yield insight at the molecular level into how different cell fate decisions can be triggered in somatic cells by the same reprogramming factors.

We have applied our approach successfully to generate hUiNPCs from three individuals at ages 10 , 25 and 37 years, with an average reprogramming efficiency around 0.2\% (Supplementary Fig. 1). We envision that our protocols can be further applied to HUCs isolated from patients with neural disorders such as Parkinson's disease, Alzheimer's disease or other neurodegenerative diseases. These patient-specific hUiNPCs should be useful for modeling disease and for drug screening. hUiNPCs may in the future also prove useful for cell therapy, such as for patients with spinal cord injury. Our feeder- and serum-free culture system for generating these cells can enable this application. The overall efficiency of $\sim 0.2 \%$ for the generation of hUiNPCs from HUCs is operationally adequate for current research, although future improvements to efficiency could be made, as has been accomplished for iPSC generation 41 .

\section{Acknowledgments}

We thank M. Esterban for helpful suggestions, Z. Li for providing support in the initial phase of this work and members of our labs for their kind help. This work is supported by the Strategic Priority Research Program of the Chinese Academy of Sciences (grant nos. XDA01020202 and XDA01020401); National Basic Research Program of China, 973 Program of China (2012CB966503 and 2012CB966802); National S\&T Major Special Project on Major New Drug Innovation (2011ZX09102-010); and National Natural Science Foundation of China (31200970 and 91213304). D.P. and G.P. are supported by the 100 Talents Project of Chinese Academy of Sciences, China.

\section{Author contributions}

G.P., Lihui, W. and D.P. conceived hypotheses and designed the experiments. Lihui, W. and W.H. performed the experiments and generated data in all figures. In addition, Linli, W., D.Q., Y.X. and Z.S. performed experiments for Figure 1 and Supplementary Figure 2; H.S., W.W. and K.F.S. participated in experiments and analysis for Figure 5; X.B. provided reagents and experimental assistance for miR302-367; B.L. performed the experiments for Figure 1; and H.W. and J.H. performed the experiments for Figure 4. G.P. and D.P. wrote the paper.

1. Aboody, K., Capela, A., Niazi, N., Stern, J.H. \& Temple, S. Translating stem cell studies to the clinic for CNS repair: current state of the art and the need for a Rosetta stone. Neuron 70, 597-613 (2011).

2. Breunig, J.J., Haydar, T.F. \& Rakic, P. Neural stem cells: historical perspective and future prospects. Neuron 70, 614-625 (2011).

3. Takahashi, K. \& Yamanaka, S. Induction of pluripotent stem cells from mouse embryonic and adult fibroblast cultures by defined factors. Cell 126, 663-676 (2006).

4. Yu, J. et al. Induced pluripotent stem cell lines derived from human somatic cells. Science 318, 1917-1920 (2007).

5. Takahashi, K. et al. Induction of pluripotent stem cells from adult human fibroblasts by defined factors. Cell 131, 861-872 (2007).

6. Hu, B.Y. et al. Neural differentiation of human induced pluripotent stem cells follows developmental 
principles but with variable potency. Proc. Natl. Acad. Sci. USA 107, 4335-4340 (2010).

7. Park, I.H. et al. Disease-specific induced pluripotent stem cells. Cell 134, 877-886 (2008).

8. Soldner, F. et al. Generation of isogenic pluripotent stem cells differing exclusively at two early onset Parkinson point mutations. Cell 146, 318-331 (2011).

9. Israel, M.A. et al. Probing sporadic and familial Alzheimer's disease using induced pluripotent stem cells. Nature 482, 216-220 (2012).

10. Vierbuchen, T. et al. Direct conversion of fibroblasts to functional neurons by defined factors. Nature $\mathbf{4 6 3}$, 1035-1041 (2010).

11. Yoo, A.S. et al. MicroRNA-mediated conversion of human fibroblasts to neurons. Nature 476, 228-231 (2011).

12. Caiazzo, M. et al. Direct generation of functional dopaminergic neurons from mouse and human fibroblasts. Nature 476, 224-227 (2011).

13. Ambasudhan, R. et al. Direct reprogramming of adult human fibroblasts to functional neurons under defined conditions. Cell Stem Cell 9, 113-118(2011).

14. Son, E.Y. et al. Conversion of mouse and human fibroblasts into functional spinal motor neurons. Cell Stem Cell 9, 205-218 (2011).

15. Qiang, L. et al. Conversion of mouse and human fibroblasts into functional spinal motor neurons. Cell 146, 359-371 (2011).

16. Huang, P. et al. Induction of functional hepatocyte-like cells from mouse fibroblasts by defined factors. Nature 475, 386-389 (2011).

17. Sekiya, S. \& Suzuki, A. Direct conversion of mouse fibroblasts to hepatocyte-like cells by defined factors. Nature 475, 390-393 (2011).

18. Zhou, Q., Brown, J., Kanarek, A., Rajagopal, J. \& Melton, D.A. In vivo reprogramming of adult pancreatic exocrine cells to $\beta$-cells. Nature 455, 627-632 (2008).

19. Zhou, T. et al. Generation of induced pluripotent stem cells from urine. J. Am. Soc. Nephrol. 22, 1221-1228 (2011).

20. Yu, J. et al. Human induced pluripotent stem cells free of vector and transgene sequences. Science 324, 797-801 (2009).

21. Liao, B. et al. MicroRNA cluster 302-367 enhances somatic cell reprogramming by accelerating a mesenchymal-to-epithelial transition. J. Biol. Chem. 286, 17359-17364 (2011).

22. Ludwig, T.E. et al. Feeder-independent culture of human embryonic stem cells. Nat. Methods 3, 637-646 (2006).

23. Ying, Q.L. et al. The ground state of embryonic stem cell self-renewal. Nature 453, 519-523 (2008).

24. Shi, Y. et al. A combined chemical and genetic approach for the generation of induced pluripotent stem cells. Cell Stem Cell 2, 525-528 (2008).

25. Lluis, F. et al. Periodic activation of Wnt/ $\beta$-catenin signaling enhances somatic cell reprogramming mediated by cell fusion. Cell Stem Cell 3, 493-507 (2008).

26. Watanabe, K. et al. A ROCK inhibitor permits survival of dissociated human embryonic stem cells. Nat. Biotechnol. 25, 681-686 (2007).

27. Burdon, T., Stracey, C., Chambers, I., Nichols, J. \& Smith, A. Suppression of SHP-2 and ERK signalling promotes self-renewal of mouse embryonic stem cells. Dev. Biol. 210, 30-43 (1999).

28. Yu, J., Chau, K.F., Vodyanik, M.A., Jiang, J. \& Jiang, Y. Efficient feeder-free episomal reprogramming with small molecules. PLoS ONE 6, e17557 (2011).

29. Hao, J. et al. In vivo structure-activity relationship study of dorsomorphin analogues identifies selective VEGF 
and BMP inhibitors. ACS Chem. Biol. 5, 245-253 (2010).

30. Kim, J. et al. Direct reprogramming of mouse fibroblasts to neural progenitors.

Proc. Natl. Acad. Sci. USA 108, 7838-7843 (2011).

31. Reynolds, B.A. \& Weiss, S. Generation of neurons and astrocytes from isolated cells of the adult mammalian central nervous system. Science 255, 1707-1710 (1992).

32. Zhang, X. et al. Pax6 is a human neuroectoderm cell fate determinant. Cell Stem Cell 7, 90-100 (2010).

33. Liu, H. \& Zhang, S.C. Specification of neuronal and glial subtypes from human pluripotent stem cells. Cell. Mol. Life Sci. 68, 3995-4008 (2011).

34. Hu, B.Y., Du, Z.W., Li, X.J., Ayala, M. \& Zhang, S.C. Human oligodendrocytes from embryonic stem cells: conserved SHH signaling networks and divergent FGF effects. Development 136, 1443-1452 (2009).

35. Thier, M. et al. Direct conversion of fibroblasts into stably expandable neural stem cells. Cell Stem Cell 10, 473-479 (2012).

36. Ring, K.L. et al. Direct reprogramming of mouse and human fibroblasts into multipotent neural stem cells with a single factor. Cell Stem Cell 11, 100-109 (2012).

37. Han, D.W. et al. Direct reprogramming of fibroblasts into neural stem cells by defined factors. Cell Stem Cell 10, 465-472 (2012).

38. Sheng, C. et al. Direct reprogramming of Sertoli cells into multipotent neural stem cells by defined factors. Cell Res. 22, 208-218 (2012).

39. Buganim, Y. et al. Single-cell expression analyses during cellular reprogramming reveal an early stochastic and a late hierarchic phase. Cell 150, 1209-1222 (2012).

40. Pan, G. \& Pei, D. Order from chaos: single cell reprogramming in two phases. Cell Stem Cell 11, 445-447 (2012).

41. Esteban, M.A. et al. Vitamin C enhances the generation of mouse and human induced pluripotent stem cells. Cell Stem Cell 6, 71-79 (2010).

\section{Methods}

Isolation and culture of HUCs. Three donors were recruited for urine samples with informed consent based on IRB approval (no. GIBH-IRB02-2009002) at Guangzhou Institutes of Biomedicine and Health (GIBH). The procedures and purposes for isolating urine cells and generating stem cells were explained to the donors in detail, and questions, if any, were answered in full. We then obtained a formal signed consent form and collected a total of $\sim 500 \mathrm{ml}$ of urine from each donor. The subsequent procedures for isolating human urine cells and generating stem cells were performed as approved. The method for isolating urine cells was modified from a previous established protocol19. Briefly, urine samples were collected at the mid-stream from three individuals and centrifuged to collect the exfoliated cells. The primary urine cells were then processed and cultured in urine cell medium consisting of a 1:1 mixture of DMEM/F12 culture medium supplemented with 10\% of FBS (FBS, PAA), $0.1 \mathrm{mM}$ non-essential amino acids (NEAA), $1 \mathrm{mM}$ GlutaMAX (Life Technologies), $0.1 \mathrm{mM} \beta$-mercaptoethanol and SingleQuot Kit CC-4127 REGM (Lonza).

hUiNPC generation. For reprogramming, an oriP/EBNA1-based pCEP4 episomal vector containing the OCT4, SOX2, KLF4 and SV4OLT genes20 and a pCEP4 vector carrying the miR302-367 precursor21 were co-transfected into urine cells via nucleofection (Amaxa Basic Nucleofector Kit for primary mammalian epithelial cells with the T-013 program, Lonza). 
Transfected urine cells were directly plated to Matrigel-coated six-well plates $(1-3 \times 105$ cells per well) in urine cell culture medium. On day 2 post-transfection, the media were changed into reprogramming media mTeSR or $5 \mathrm{i}$ (mTeSR supplemented with $5 \mathrm{i} 0.5 \mu \mathrm{M}$ A83-01, $1 \mu \mathrm{M}$ PD0325901, $3 \mu \mathrm{M}$ CHIR99021, $0.5 \mu \mathrm{M}$ thiazovivin and $0.2 \mu \mathrm{M}$ DMH1). Medium was changed every $2 \mathrm{~d}$ during the reprogramming. Fifteen days after transfection, colonies were picked up in $5 \mathrm{i}$ and transferred onto a new Matrigel plate. For further passaging, the cells were dissociated to small clusters or single cells for suspension in flasks containing neural growth medium as neural spheres (1:1 of DMEM/F12 supplemented with 1\% N2 (Invitrogen) and Neurobasal medium supplemented with 2\% B27 (Invitrogen) supplemented with $20 \mathrm{ng} / \mathrm{ml} \mathrm{bFGF} \mathrm{and} 20 \mathrm{ng} / \mathrm{ml} \mathrm{EGF).}$

Neural differentiation in vitro. hUiNPCs were cultured in neural medium N2B27 containing EGF and bFGF. For pan-neuronal differentiation, hUiNPC spheres were plated on Matrigel-coated coverslips and cultured in neural N2B27 medium with the withdrawal of EGF and bFGF and the addition of neurotrophic factors, BDNF, GDNF, CNTF, IGF1 (all at $10 \mathrm{ng} / \mathrm{ml}$, Peprotech) and $1 \mu \mathrm{M}$ cAMP to improve neuronal survival. hUiNPCs differentiated for 2 weeks were then examined for the expression of neuronal markers and an astrocyte marker. To derive oligodendrocytes, hUiNPCs were plated on poly-l-ornithine/laminin substrate and cultured in DMEM/F12 supplemented with 1\% N1 (Sigma), biotin (100 ng/ml), PDGF-AA (20 ng/ml, Peprotech), NT3 (20ng/ml, Peprotech), $1 \mu \mathrm{M}$ cAMP and bFGF (10 ng/ml) for 1 week. Afterward, bFGF was replaced by IGF1 $(10 \mathrm{ng} / \mathrm{ml})$ for another 3 weeks. All media were replenished every 2 d.

Quantitative real-time PCR. Total RNAs were extracted with Trizol (Invitrogen). qRT-PCR was performed using a Thermal Cycler Dice Real Time System and SYBR Premix EX Taq

(Takara). $\beta$-actin was used for qRT-PCR normalization, and all data were measured in triplicate. Primer sequences are listed in Supplementary Table 3.

Analysis of gene integration by PCR. Genomic DNAs were extracted using the Wizard Genomic DNA Purification Kit (Promega) for PCR analysis using primers (Supplementary Table 3) that specifically amplify the exogenous transgenes as described 20 .

Immunocytochemistry. Cells were fixed in $4 \%$ paraformaldehyde dissolved in $0.1 \mathrm{M}$ phosphate buffer (PB) for $20 \mathrm{~min}$. After several washes with $0.01 \mathrm{M}$ phosphate-buffered saline (PBS), the cultures were incubated with the primary antibodies in PBS plus $1 \%$ BSA, $10 \%$ normal goat serum and $0.3 \%$ Triton $\mathrm{X}-100$ overnight at $4{ }^{\circ} \mathrm{C}$. The primary antibodies are listed in Supplementary Table 4. Primary antibodies were visualized with species-specific secondary antibody conjugated to the fluorescent labels Alexa 568 or 488 (1:400; Invitrogen). Cells were mounted in anti-fade medium containing 4',6-diamidino-2-phenylindole (Sigma) to counterstain nuclei. At least 500 DAPI-positive cells from five randomly selected fields were counted to calculate the fraction of each lineage. Results are mean \pm s.e.m. of data from three experiments unless stated otherwise in legends. Cells were imaged on a Zeiss Axio Imager A1 microscope or a Leica TCS SP2 Spectral confocal microscope.

Electrophysiological analysis. Whole-cell patch-clamp recording techniques were used to study the physiological properties of hUiNPC-derived neurons in culture with borosilicate glass pipettes (resistance 5-10 M $\Omega$ ) using an Axopatch 200B amplifier (Axon Instruments for Molecular 
Devices). Both the spontaneous postsynaptic current and current response to exogenous focal application of glutamate and GABA were recorded. Pressure ejection was used to puff $1 \mathrm{mM}$ glutamate (10 p.s.i., $100 \mathrm{~ms}$ ) and $1 \mathrm{mM} \mathrm{GABA}(10$ p.s.i., $100 \mathrm{~ms})$, and the holding voltages were $-80 \mathrm{mV}$ and $0 \mathrm{mV}$, respectively. The patch pipette internal solution contained (in $\mathrm{mM}$ ): 136.5 K-gluconate, 0.2 EGTA, 10 HEPES, $9 \mathrm{NaCl}, 17.5 \mathrm{KCl}, 4 \mathrm{Mg}$-ATP and $0.3 \mathrm{Na}$-GTP, adjusted with $\mathrm{KOH}$ to $\mathrm{pH} 7.2,285 \mathrm{osmol} / \mathrm{l}$. For the recording of voltage-gated currents and action potentials, we used the following composition of the intracellular solution (in $\mathrm{mM}$ ): 140 potassium methanesulfonate, 10 HEPES, 5 NaCl, 1 CaCl2, 0.2 EGTA, 3 ATP-Na2, 0.4 GTP-Na2, pH 7.3 (adjusted with $\mathrm{KOH}$ ). The external solution contained (in $\mathrm{mM}$ ): $120 \mathrm{NaCl}, 1.2 \mathrm{KH} 2 \mathrm{PO} 4,1.9 \mathrm{KCl}$, $26 \mathrm{NaHCO} 3,2.2 \mathrm{CaCl} 2,1.4 \mathrm{MgSO} 4,10$ d-glucose, 7.5 HEPES (pH with $\mathrm{NaOH}$ to 7.3). The bath solution was equilibrated with 95\% $\mathrm{O} 2$ and 5\% $\mathrm{CO} 2$ before use. Resting potentials were maintained at about $-60 \mathrm{mV}$. Signals were sampled at $10 \mathrm{kHz}$ using a Digidata1440A analog-to-digital converter and acquired and stored on a computer hard drive using pClamp10 software. Data were analyzed using pClamp10 (Clampfit).

Karyotype analysis. hUiNPCs were used for karyotype analysis as described42. Cells were grown in 10-cm plates, and demecolcine (Dahui Biotech) was added to a final concentration of 50 $\mu \mathrm{g} / \mathrm{ml}$ for $40 \mathrm{~min}$. Cells were then trypsinized, pelleted by centrifugation at $200 \mathrm{~g}$ for $5 \mathrm{~min}$, resuspended in $8 \mathrm{ml}$ of $0.075 \mathrm{M} \mathrm{KCl}$ and incubated for $20 \mathrm{~min}$ at $37{ }^{\circ} \mathrm{C}$. Fixative solution composed of one part acetic acid and three parts methanol was added to a final

volume of $10 \mathrm{ml}$, mixed gently and incubated for $10 \mathrm{~min}$ at $37{ }^{\circ} \mathrm{C}$. After further centrifugation, the supernatant was removed, and ice-cold fixative solution composed of one part acetic acid and three parts methanol was added to a final volume of $10 \mathrm{ml}$. Cells were dropped on a cold slide and incubated at $75{ }^{\circ} \mathrm{C}$ for $3 \mathrm{~h}$. Belts were treated with trypsin and colorant, and metaphase states were analyzed on an Olympus BX51 microscope.

Whole-genome expression analysis. Total RNAs were extracted from HUCs, hUiNPCs and Uri_iPSCs and quantified by the NanoDrop ND-1000. RNA integrity was assessed by standard denaturing agarose gel electrophoresis. About $5 \mu \mathrm{g}$ total RNA was used for the reverse transcription with Invitrogen's Superscript Double-Stranded cDNA Synthesis Kit. Labeled cDNA was synthesized by in vitro transcription using NimbleGen one-color DNA labeling kit. Array hybridization was performed with the NimbleGen Hybridization System and followed by a wash with the NimbleGen wash buffer kit. Arrays were scanned with the Axon GenePix 4000B microarray scanner. Data were analyzed with NimbleScan software (v.2.5).

In vivo transplantation and tissue processing. The protocol used for newborn rat was reviewed and approved by the animal care committee at GIBH. For hUiNPCs transplantation, 12 newborn Sprague Dawley rats were used in the study. hUiNPC neurospheres were gently dissociated into single cells with Accutase (Millipore) and resuspended in N2 medium supplemented with 10 $\mu \mathrm{g} / \mathrm{ml} \mathrm{BDNF}, 10 \mu \mathrm{g} / \mathrm{ml}$ GDNF, $10 \mu \mathrm{g} / \mathrm{ml}$ CNTF and $10 \mu \mathrm{g} / \mathrm{ml}$

42. Esteban, M.A. et al. Generation of induced pluripotent stem cell lines from Tibetan miniature pig. J. Biol. Chem. 284, 17634-17640 (2009).

IGF1 at a concentration of $1.0 \times 105$ cells $/ \mu 1$ and placed on ice for the duration of the grafting session. One microliter of cell suspension was slowly injected into each of the striatum of cryoanesthetized newborn rats through a Hamilton syringe with the sharpened tip. At 2 and 4 
weeks following transplantation, rats were anesthetized and perfused intracardially with $0.01 \mathrm{M}$ PBS, pH 7.4, followed by 100-200 ml of fixative solution containing $4 \%$ paraformaldehyde in 0.1 $\mathrm{M}$ PBS, $\mathrm{pH}$ 7.4. The brains were harvested and post-fixed in fresh fixative solution overnight and subsequently placed in $30 \%$ sucrose, $0.1 \mathrm{M} \mathrm{PBS}$ at $4{ }^{\circ} \mathrm{C}$ for $2-3 \mathrm{~d}$. The samples were then cut into $25-\mu \mathrm{m}$ cross-sections on a microtome. The serial sections were collected in 0.01 M PBS and kept at $4{ }^{\circ} \mathrm{C}$ for further study.

Immunohistochemistry of brain sections. Donor cells were identified by an antibody to a human-specific nuclear antigen (hNA). Immunopositive cells were double labeled with antibodies to $\beta$ III tubulin (TUJ1), GFAP and nestin (Supplementary Table 4). Species-specific fluorescence-conjugated secondary antibodies conjugated to Alexa 488 (1:400; Molecular Probes) were applied for $2 \mathrm{~h}$ at $20{ }^{\circ} \mathrm{C}$. Sections were then counterstained with 4',6-diamidino-2-phenylindole to stain nuclei and coverslipped with anti-fade mounting medium (FluorSave; Calbiochem). A Zeiss 710 NLO spectral confocal microscope was used for all immunofluorescence studies. 


\section{Figure 1}
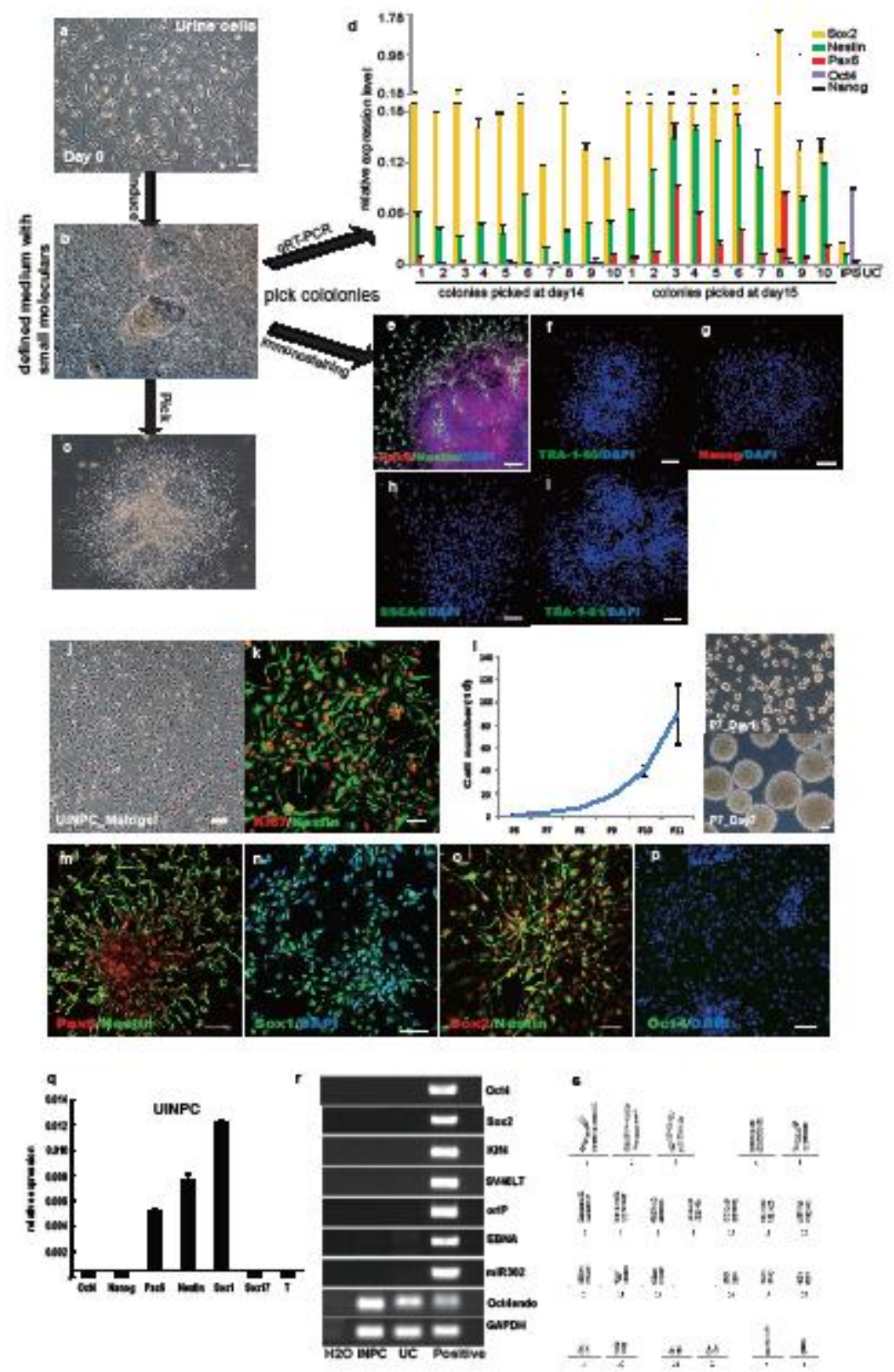

Figure 1 | Generation and expansion of integration-free NPCs from human urine cells. (a-d) Generation of NPCs from human urine cells. Epithelial-like cells isolated from human urine (a) were transfected with episomal vectors encoding reprogramming factors and miR302-367 and cultured in defined medium (5i) on Matrigel. Colonies arising at day 12 (b) were picked and re-plated onto Matrigel (c) or directly analyzed for marker expression by qRT-PCR (d). The plot shows relative expression levels of the indicated genes in colonies picked at days 14 and 
15 compared to levels in HUCs and iPSCs (abbreviated as UC and iPS, respectively). Note that levels of NANOG in iPSCs are invisible on the plot at this scale. The value of NANOG in iPSCs was 0.0061, compared with 0.0000036 in HUCs and 0.00165 in colonies at day 14 and 0.000899 in colonies at day 15. (e-i) Micrographs show immunostains of colonies for the indicated markers (day 15). Lower panel of $\mathbf{g}$ shows a positive control for the antibody to NANOG. (j-q) Expansion of hUiNPCs. Representative morphology of hUiNPCs cultured on Matrigel (j) or in suspension as neural spheres (k) is shown. (k) Left, growth curve of hUiNPCs cultured as neural spheres. Right, neural spheres before and after passage (P). (l) hUiNPCs stained for Ki67 and nestin (P6). (m-p) Immunostains of expanded hUiNPCs (P5) for the indicated markers. (q) qRT-PCR analysis of markers expressed by expanded hUiNPCs (P5). (r) PCR detection of integrated transgenes in expanded hUiNPCs (iNPC, P6). Primers were designed to detect exogenous reprogramming factors. Untransfected urine cells served as the negative control. HUCs transiently transfected with reprogramming factors served as the positive control. (s) Karyotype of expanded hUiNPCs (P5). Scale bars, $50 \mu \mathrm{m}$. Error bars, s.d., based on three replicates $(n=3)$ for $\mathbf{d}, \mathbf{k}$ and $\mathbf{q}$. 


\section{Figure 2}

a

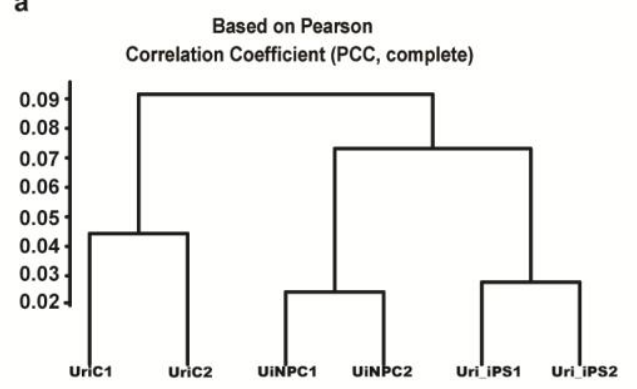

b

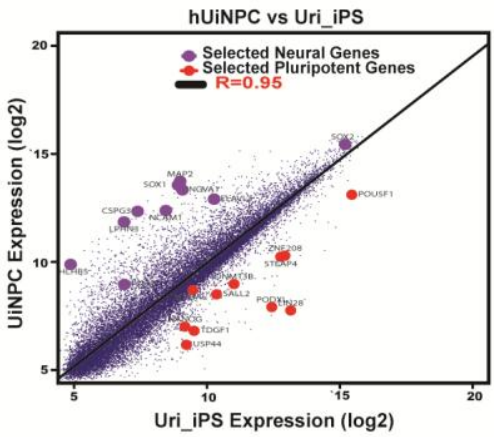

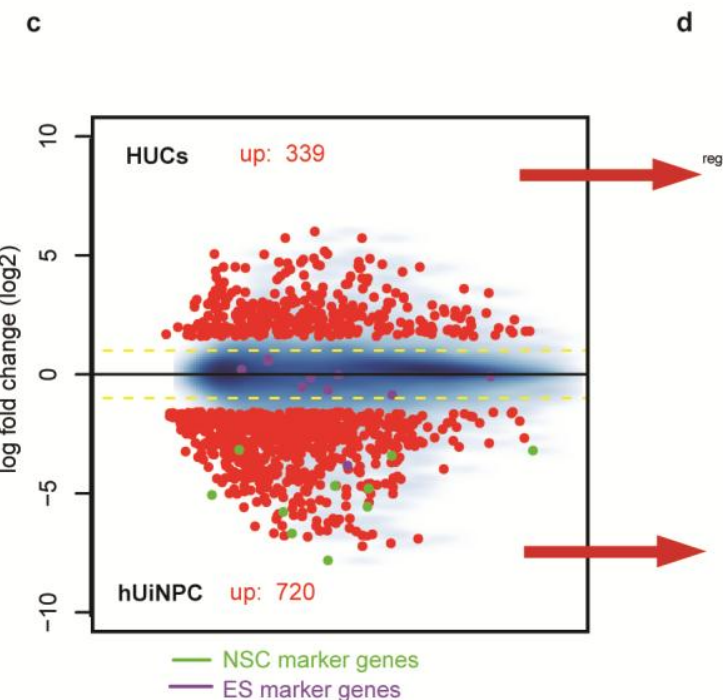

d
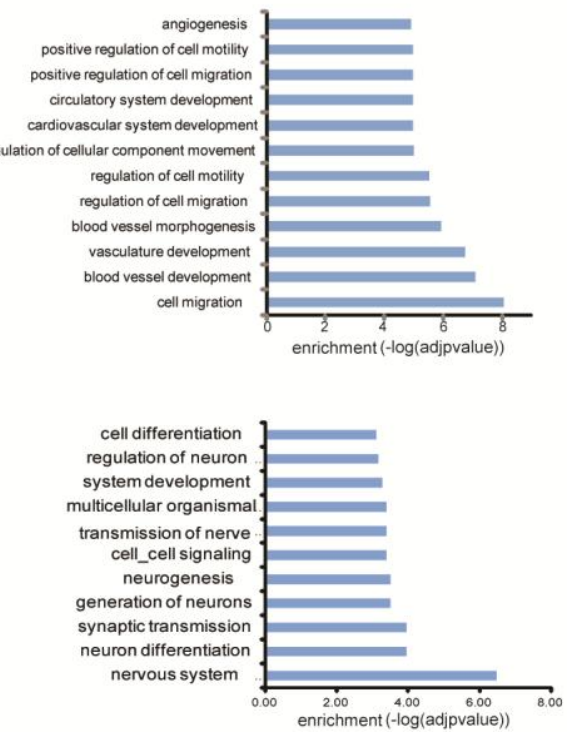

Figure 2 | Global gene expression analysis of hUiNPCs. (a) Pearson correlation analyses of global gene expression in HUCs (UriC1 and UriC2) and in neural progenitor cells (UiNPC1 and UiNPC2) and iPSCs (Uri_iPSC1 and Uri_iPSC2) derived from them. (b) Comparison of global gene expression profiles of a Uri_iPSC and UiNPC line derived from the same starting cell sample. $R$, Pearson correlation coefficient. Selected neural-specific (purple) and embryonic stem cell (ESC)-specific (red) genes are highlighted. (c) Differential expression profile between parental HUCs (above) and hUiNPCs (below). Yellow dashed lines correspond to a twofold change. The differentially expressed genes (red) are those with an adjusted $P$ value 0.05 and fold change 3 . Known NPC-specific (green) and ESC-specific (purple) genes are depicted. (d) Functional annotations of genes differentially expressed between HUCs (top) and hUiNPCs (bottom). Gene ontology (GO) statistics for these genes were computed using the hypergeometric test, and enriched GO terms (biological processes) for each cell type are plotted with $-\log _{10}$ of the adjusted $P$ values (adjpvalue). 


\section{Figure 3}
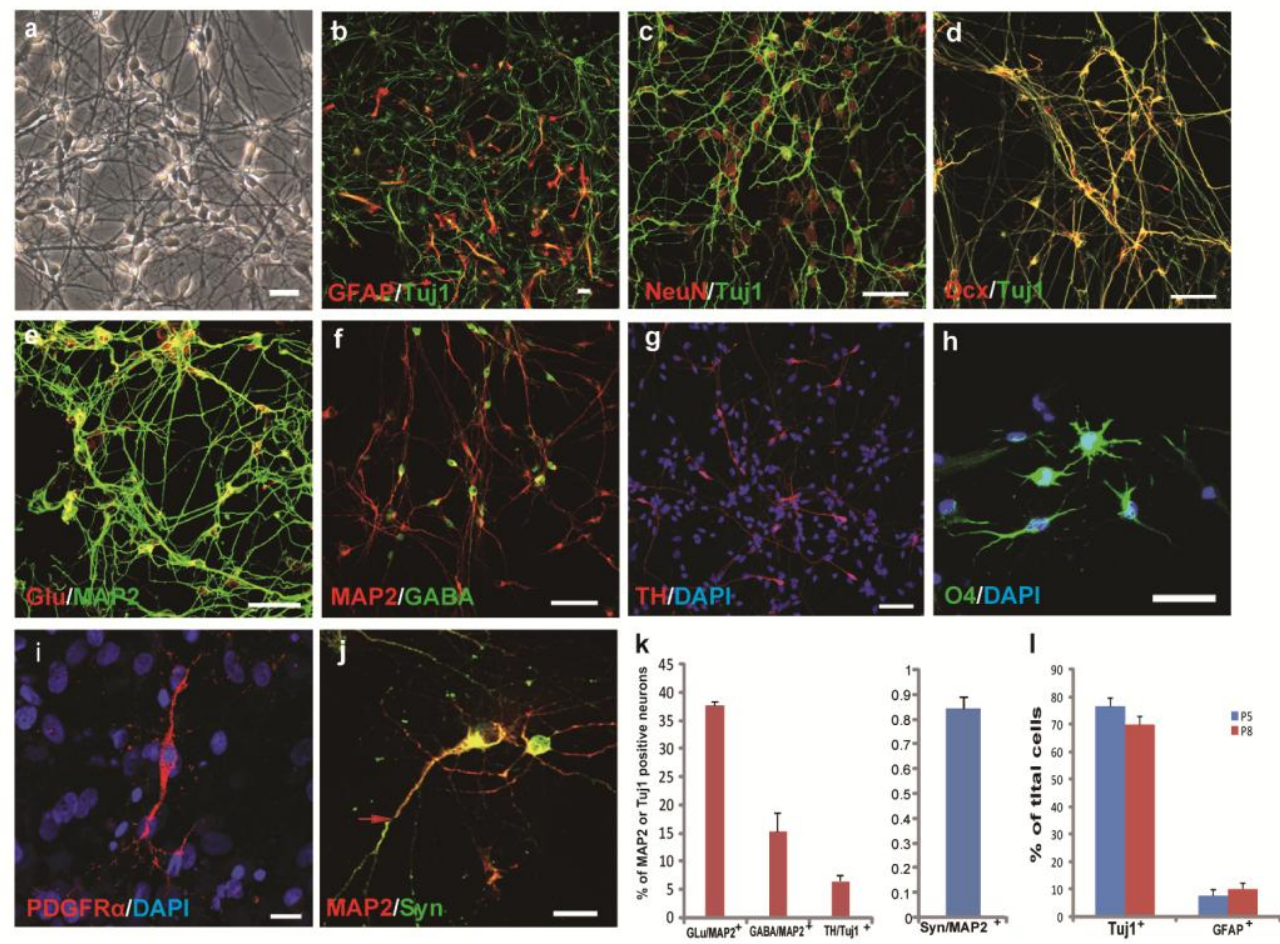

Figure 3 | Differentiation of hUiNPCs in vitro. (a) Bright-field image of spontaneously differentiated cells from hUiNPCs. (b-j) Immunostains of spontaneously differentiated hUiNPCs with antibodies against the indicated markers: GFAP, astrocyte marker; TUJ1, pan-neuronal marker; NeuN and MAP2, mature neuronal markers; DCX, immature neuronal marker; glutamate (Glu), GABA and TH, subtype-specific neuronal markers; O4, oligodendrocyte marker; PDGFR- $\alpha$, oligodendrocyte progenitor marker; SYN (and arrow in $\mathbf{j}$ ), synapsin I. (k) Percentage of each neuronal subtype (left) and of neurons with synapsin staining (right) out of total neurons. Total neurons were determined by staining with pan-neuronal marker MAP2 or TUJ1. At least 500 DAPI-positive cells from five randomly selected fields were counted to calculate the fraction of each lineage (Online Methods). (I) Percentage of neurons (TUJ1-positive cells) and astrocytes (GFAP-positive cells) in spontaneously differentiated hUiNPCs at different passages (P). Total cells were determined by DAPI stain. Scale bars: $50 \mu \mathrm{m}(\mathbf{a}-\mathbf{g}), 20 \mu \mathrm{m}$ $(\mathbf{h}-\mathbf{j})$. Error bars, s.e.m.; $n=3$ experiments. 


\section{Figure 4}

a
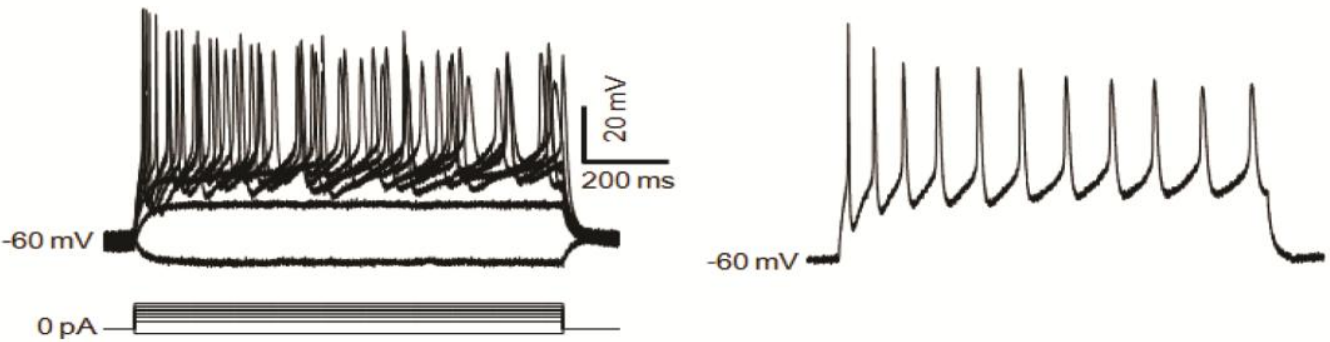

b

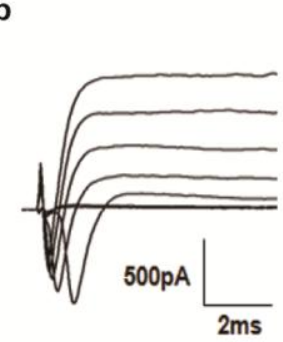

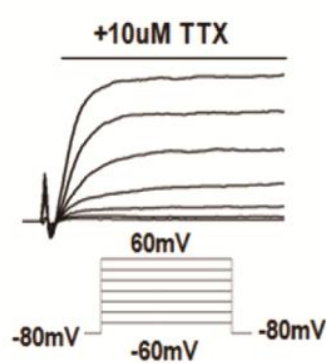

d

Spontaneous PSCs c
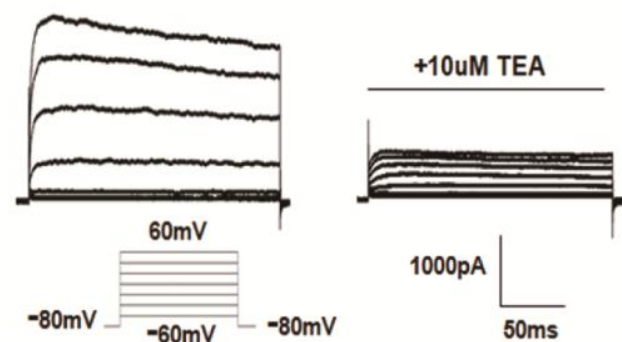
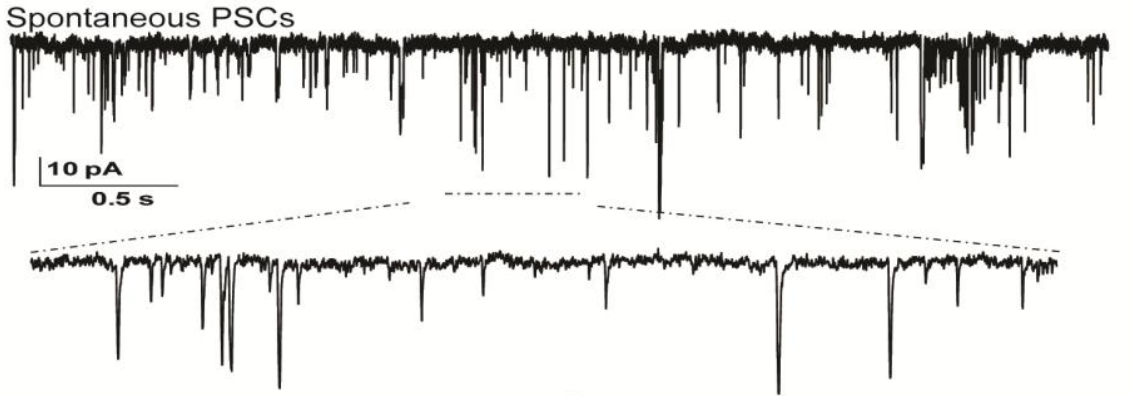

e
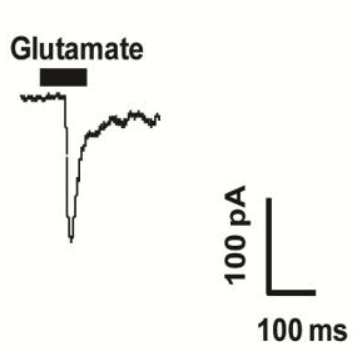

f

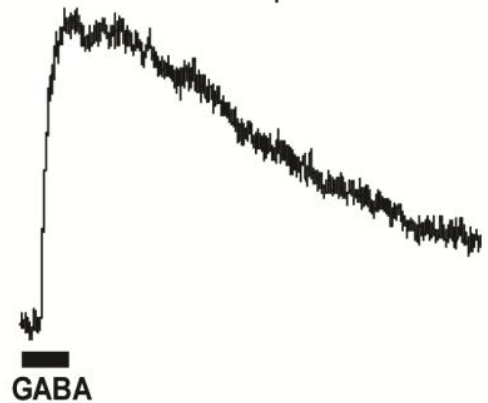

Figure 4 | hUiNPC-derived neurons are functional in vitro. (a) Current-clamp recording showing a representative train of action potentials in a neuron differentiated from hUiNPCs. Intracellular injected currents were step currents of -20, 30, 50, 60, 70, 80, 90 and 100 pA. Right, current trace at 90-pA injected current. (b,c) Representative recordings of voltage-gated ion channels from a neuron differentiated from hUiNPCs. An inward current was observed and could be blocked by tetrodotoxin (TTX) (b), and an outward current could be blocked by tetraethylammonium (TEA) (c). (d-f) Analysis of postsynaptic currents in hUiNPC-derived neurons. Shown are representative traces of spontaneous postsynaptic currents (PSCs) (d), of excitatory postsynaptic currents in cells clamped at $-80 \mathrm{mV}$ in response to 1-glutamate puffs (e), and of inhibitory postsynaptic currents in cells clamped at $0 \mathrm{mV}$ in response to GABA puffs (f). 


\section{Figure 5}

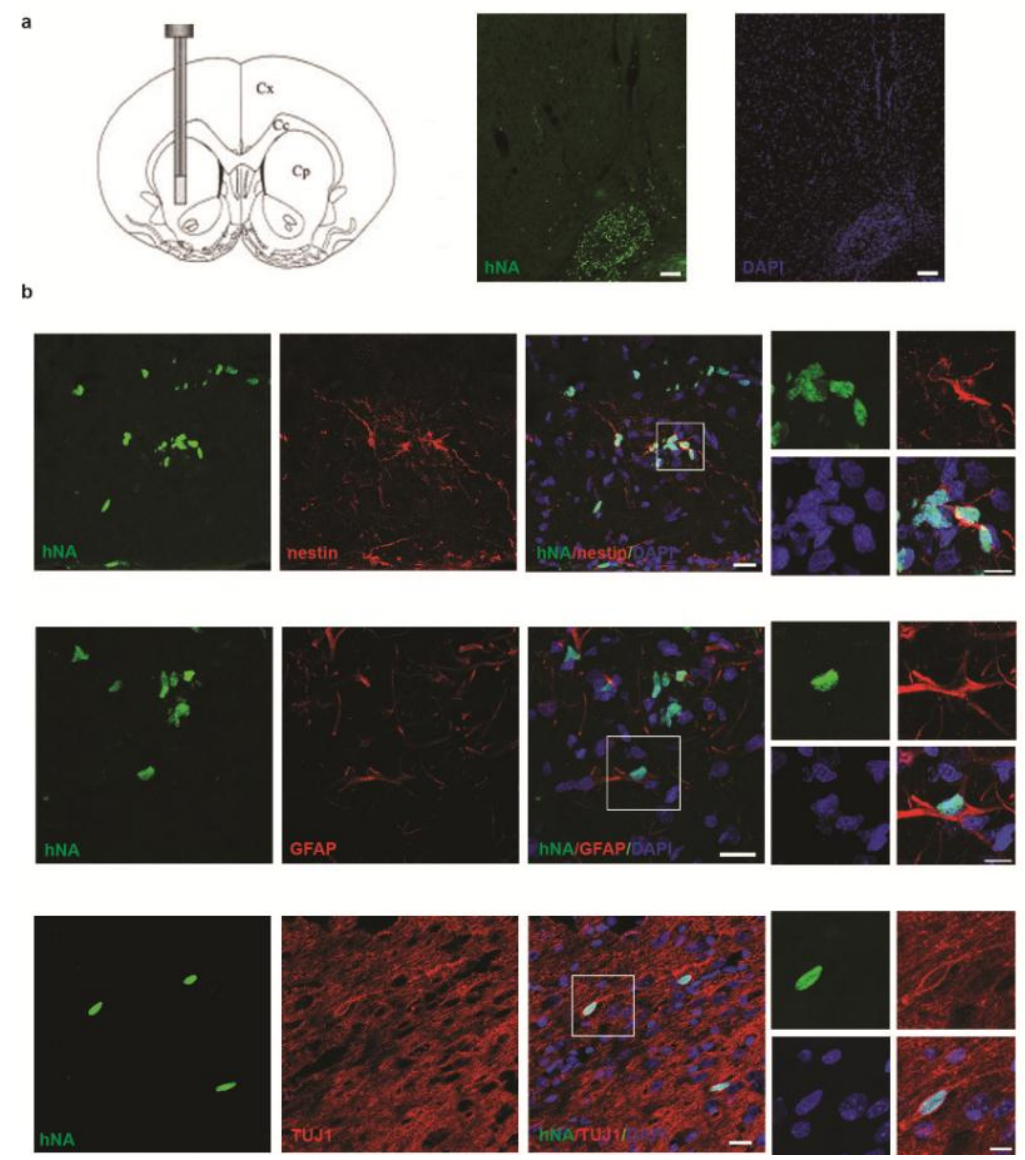

Figure 5 | In vivo transplant of hUiNPCs. (a) Schematic shows the transplant location (striatum) of hUiNPCs into the brain of newborn rats. Micrographs show immunostains for human nuclear-specific antigen (hNA) and DAPI stain on a brain section at the transplant site. (b) Immunostains for the indicated markers of brain sections at the transplant site 4 weeks after cells were transplanted. Scale bars: $50 \mu \mathrm{m}(\mathbf{a}), 20 \mu \mathrm{m}(\mathbf{b}$, left), $10 \mu \mathrm{m}(\mathbf{b}$, right). 